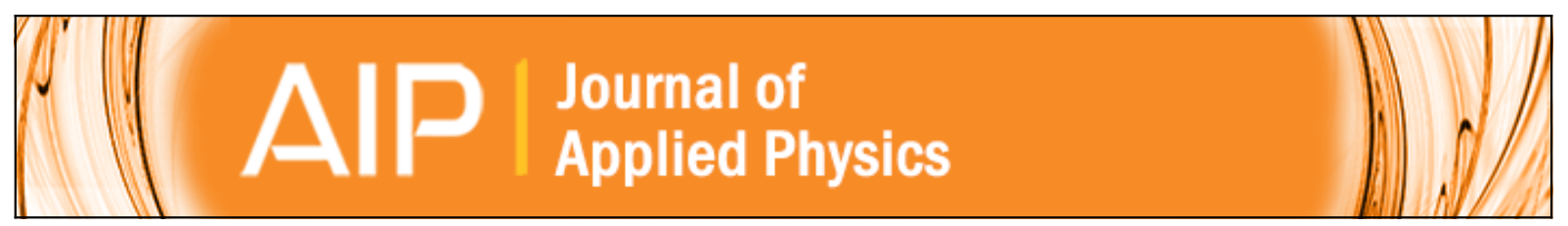

\title{
Off-axis electron holography of ferromagnetic multilayer nanowires
}

Azadeh Akhtari-Zavareh, L. P. Carignan, A. Yelon, D. Ménard, T. Kasama, R. Herring, R. E. Dunin-Borkowski, M. R. McCartney, and K. L. Kavanagh

Citation: Journal of Applied Physics 116, 023902 (2014); doi: 10.1063/1.4887488

View online: http://dx.doi.org/10.1063/1.4887488

View Table of Contents: http://scitation.aip.org/content/aip/journal/jap/116/2?ver=pdfcov

Published by the AIP Publishing

\section{Articles you may be interested in}

Ferromagnetic resonance in soft-magnetic metallic glass nanowire and microwire

Appl. Phys. Lett. 105, 202403 (2014); 10.1063/1.4902147

Structural and magnetic characterization of as-prepared and annealed FeCoCu nanowire arrays in ordered anodic aluminum oxide templates

J. Appl. Phys. 115, 133904 (2014); 10.1063/1.4870289

Electrochemical synthesis of highly ordered magnetic multilayered nanowire arrays

AIP Conf. Proc. 1455, 85 (2012); 10.1063/1.4732474

Surface magnetization processes in soft magnetic nanowires

J. Appl. Phys. 107, 09E315 (2010); 10.1063/1.3360209

Off-axis electron holography of exchange-biased CoFe/FeMn patterned nanostructures

J. Appl. Phys. 90, 2899 (2001); 10.1063/1.1390493

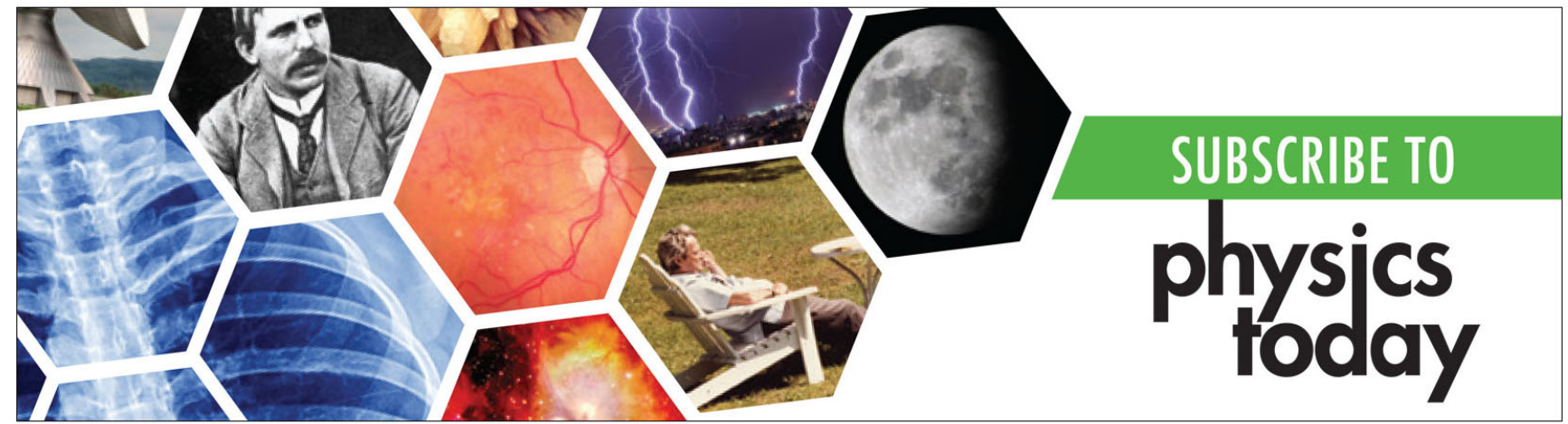




\title{
Off-axis electron holography of ferromagnetic multilayer nanowires
}

\author{
Azadeh Akhtari-Zavareh, ${ }^{1}$ L. P. Carignan,,${ }^{2,3,4}$ A. Yelon, ${ }^{3}$ D. Ménard, ${ }^{3}$ T. Kasama, ${ }^{5}$ \\ R. Herring, ${ }^{6}$ R. E. Dunin-Borkowski, ${ }^{7}$ M. R. McCartney, ${ }^{8}$ and K. L. Kavanagh ${ }^{1}$ \\ ${ }^{1}$ Department of Physics, Simon Fraser University, Burnaby, British Columbia V5A1S6, Canada \\ ${ }^{2}$ Apollo Microwaves, 1650 Trans-Canada Highway, Dorval, Quebec H9P 1H7, Canada \\ ${ }^{3}$ Department of Engineering Physics, École Polytechnique de Montréal, Montréal, Quebec, H3C 3 A7 Canada \\ ${ }^{4}$ Department of Electrical Engineering, École Polytechnique de Montréal, Montréal, Quebec, H3C 3A7 Canada \\ ${ }^{5}$ Center for Electron Nanoscopy, Technical University of Denmark, DK-2800 Kongens Lyngby, Denmark \\ ${ }^{6}$ Department of Mechanical Engineering, University of Victoria, Victoria, British Columbia V8W 3P6, Canada \\ ${ }^{7}$ Ernst Ruska-Centre for Microscopy and Spectroscopy with Electrons and Institute for Microstructure \\ Research, D-52425 Jülich, Germany \\ ${ }^{8}$ Department of Physics, Arizona State University, Tempe, Arizona 85287-1504, USA
}

(Received 24 April 2014; accepted 26 June 2014; published online 8 July 2014)

We have used electron holography to investigate the local magnetic behavior of isolated ferromagnetic nanowires (NWs) in their remanent states. The NWs consisted of periodic magnetic layers of soft, high-saturation magnetization $\mathrm{CoFeB}$ alloys, and non-magnetic layers of $\mathrm{Cu}$. All NWs were fabricated by pulsed-potential electrodeposition in nanoporous alumina membranes. The NW composition and layer thicknesses were measured using scanning transmission electron microscopy and energy dispersive spectroscopy. The magnetization of individual NWs depended upon the thicknesses of the layers and the direction of an external magnetic field, which had been applied in situ. When the $\mathrm{CoFeB}$ was thicker than the diameter $(50 \mathrm{~nm})$, magnetization was axial for all external field directions, while thinner layers could be randomized via a perpendicular field. In some cases, magnetization inside the wire was detected at an angle with respect to the axis of the wires. In thinner $\mathrm{Cu} / \mathrm{CoFeB}(<10 \mathrm{~nm}$ each) multilayer, magnetic field vortices were detected, associated with opposing magnetization in neighbouring layers. The measured crystallinity, compositions, and layer thicknesses of individual NWs were found to be significantly different from those predicted from calibration growths based on uniform composition NWs. In particular, a significant fraction of $\mathrm{Cu}$ (up to 50 at. \%) was present in the $\mathrm{CoFeB}$ layers such that the measured magnetic induction was lower than expected. These results will be used to better understand previously measured effective anisotropy fields of similar NW arrays. (C) 2014 AIP Publishing LLC.

[http://dx.doi.org/10.1063/1.4887488]

\section{INTRODUCTION}

During the past two decades, one-dimensional (1D) nanomaterials have attracted considerable attention due to their important role in miniaturizing electronic devices. In particular, 1D ferromagnetic nanowires (NWs) are of interest, due to their unique physical properties, and possible application in magnetic recording, ${ }^{1,2}$ spin electronics, ${ }^{3}$ microwave materials, ${ }^{4}$ and sensor devices. ${ }^{5,6}$ Single phase or uniform composition magnetic NWs exhibit properties potentially useful for small, light, ultrahigh-density memory devices, ${ }^{7,8}$ while multilayered magnetic NWs, for which the thickness of the magnetic and non-magnetic layers can be controlled at the nanometer scale, also appear promising. ${ }^{9,10}$ In either case, the macroscopic properties of arrays of these NWs are highly dependent upon the uniformity of individual NW diameters, composition, and crystal structure.

Electrodeposition into a nanoporous alumina membrane is one method of ferromagnetic NW array fabrication. ${ }^{10}$ Electrodeposited magnetic NWs have been proposed for high frequency applications ${ }^{11}$ and for high-density storage media ${ }^{12}$ due to their relatively low cost of fabrication and the possibility of manipulating their magnetic properties by adjusting the composition and geometric parameters of the array. ${ }^{10,13}$ Electrodeposited multilayer NWs offer additional possibilities for tuning the magnetic anisotropy and magnetization response by controlling their diameter, inter-wire distance, composition, and layer thicknesses. ${ }^{4}{ }^{40}$ Understanding the effects of these geometric parameters on the magnetic remanence, the effective magnetic anisotropy, and the microwave permeability of individual and arrays of magnetic NWs is crucial to their design for a given application.

The effective magnetic anisotropy of arrays of long NWs, with small diameter and uniform composition, has been obtained by magnetometry and by ferromagnetic resonance (FMR) measurements. The results are generally well accounted for, quantitatively related to the geometric parameters, using a simple model based on the dipolar interaction between uniformly magnetized wires. ${ }^{4,10}$ In particular, the effective permeability from FMR measurements of such arrays exhibits a double resonance at small, non-saturating, fields, a behavior that was quantitatively explained by assuming two populations of mutually interacting bistable NWs. ${ }^{14}$ Under axial fields at low frequencies, large diameter (150 $\mathrm{nm}$ and above), uniform composition wires exhibit a 
linear, anhysteretic response typical of a hard axis, as predicted by the simple model. ${ }^{4,10,15}$ And small diameter $(20 \mathrm{~nm})$ uniform composition arrays exhibit rectangular hysteresis loops, typical of an easy axis, as expected. However, angle-dependent, vectorial, magnetometry of similar uniform arrays, suggested that the mechanism for magnetization reversal consists of a mixture of coherent and incoherent rotations, and that the relative importance of each mechanism is related to the wire diameters. ${ }^{15}$ These observations could not be explained by a simple model of uniformly magnetized bistable wires, with dipolar interactions only. And the agreement was only qualitative when such a model was applied to arrays of multilayer NWs. ${ }^{4,10}$

The basic structural unit in a multilayered NW is a periodic row of non-magnetic/magnetic cylinders of radius $r$. If each magnetic cylinder is of thickness, $t$, then depending upon the aspect ratio, $t / r$, the expected magnetic state may range from a vortex to a flower state. ${ }^{20}$ For large aspect ratios $(t / r>1)$, calculations indicate that the remanent magnetization should be uniform along the axis of the wire, with small radial deviations at the top and bottom. This is called an out-of-plane flower state. ${ }^{21}$ For thinner, disc-shaped, cylinders, with smaller aspect ratios $(t / r<1)$, the configuration is called an in-plane flower state. This should have a remanent magnetization nearly perpendicular to the axis of the disc, with opposite directions in alternating neighbouring discs. Magnetometry measurements of arrays of periodic $\mathrm{Cu} /$ CoFeB NWs have confirmed that their average magnetic anisotropy is a function of the thickness of each layer ${ }^{4}$ leading to a weaker anisotropy of the arrays compared with uniform $\mathrm{NWs},{ }^{4}$ as expected. However, quantitative agreement with dipolar models has not been found.

First order reversal curve (FORC) measurements ${ }^{16}$ can separate, to some extent, the intrinsic thresholds of individual magnetic elements, from their interaction with other elements. Such measurements on uniform composition NW arrays ${ }^{17,18}$ indicated that switching of an individual wire is an incoherent process, with nucleation somewhere in the wire, followed by rapid reversal of the entire wire. In the case of multilayer wires, FORC studies ${ }^{19}$ indicated that the reversal is a combination of reversible and irreversible processes. While we have speculated that discrepancies between the simple model predictions and results for multilayered NWs could be due to errors in the estimation of the layer thicknesses, composition and homogeneity, and finite interface abruptness, we have not been able to confirm such features.

Off-axis electron holography (EH) is able to study the remanent state of nanoscale magnetic materials. Combined with the conventional capabilities of scanning and transmission electron microscopy (STEM) for the characterization of composition and nanostructure, the magnetic remanent state of individual nanoscale magnets can be determined. EH analysis has already provided some insights into the magnetic properties of individual ferromagnetic NWs, including their magnetic anisotropy, interface anisotropy, and multilayer coupling as a function of their size, shape, and structure. ${ }^{21-23}$ In particular, the saturated magnetic properties of single phase (Co and $\mathrm{Ni})$ and multilayer $(\mathrm{Cu} / \mathrm{Co}) \mathrm{NWs}$ of radii ranging from 20 to
$150 \mathrm{~nm}$, and a few microns in length ${ }^{21}$ have been investigated. However, the magnetic spatial resolution in this study was limited to $70 \mathrm{~nm} .^{21} \mathrm{EH}$ has more recently been carried out on smaller single-crystalline Co wires $(4 \mathrm{~nm}$ diameters) with a spatial resolution of less than $10 \mathrm{~nm}^{22}$ The remanent magnetization state was found to be along the axis of the wire, due to the high aspect ratio, as expected.

Here, we have studied the local properties of individual NWs composed of periodic $\mathrm{Cu} / \mathrm{CoFeB}$ multilayers with various nonmagnetic/ferromagnetic (N/FM) layer thicknesses. We specifically investigated the magnetic structure of the individual layers, along with their thickness, interface abruptness, and composition, and were able to detect magnetic signals from $8 \mathrm{~nm}$ trilayer components. We have compared their individual magnetic properties to average properties of arrays of similar NWs obtained from static magnetometry measurements. ${ }^{10}$

\section{ELECTRON HOLOGRAPHY}

STEM is a powerful collection of techniques for characterizing the microstructure of materials. However, only intensity is recorded in the final images ${ }^{24,25}$ while information about the phase of the electron wave after it has traveled through the specimen is lost. EH is a unique technique that determines both the amplitude and phase shift of the electron wave via electron interference. ${ }^{26}$ This phase shift is related to the electrostatic potential of the specimen and to the component of the magnetic induction in the plane perpendicular to the electron beam. Therefore, quantitative information about magnetic and electrical properties of materials may be obtained.

In a STEM with a highly coherent electron source, such as a field emission gun (FEG), off-axis EH can be applied by positioning the area of interest of the specimen so that it covers half of the electron beam. Thus, one half of the beam passes through the specimen, while the other half is undisturbed by the specimen. An electron biprism (a fine wire) is located underneath the specimen (close to a conjugate image plane in the microscope). When sufficient voltage is applied to it, the two parts of the beam interfere at the image plane to make a hologram. If the specimen to be examined is magnetic, the off-axis EH can be carried out in a field-free environment. To do so, the conventional microscope objective lens, which has a large magnetic field (1.9 T) along the beam direction, is switched off and another lens (the so called Lorentz lens), which is below the specimen, is used for imaging. The Lorentz lens is sufficiently weak and distant from the sample that it cannot influence magnetization at the sample plane.

For a thin specimen, when dynamic diffraction can be ignored ${ }^{27}$ the electron phase as a function of position in one dimension is expressed by ${ }^{28}$

$$
\phi(x)=C_{E} \int V(x, z) d z-\frac{e}{\hbar} \iint B_{\perp}(x, z) d z d x,
$$

where $C_{\mathrm{E}}$ is an electron beam energy-dependent constant (8.24 or $7.29 \mathrm{rad} \mathrm{V}^{-1} \mu \mathrm{m}^{-1}$ at an acceleration voltage of 120 
or $200 \mathrm{kV}$, respectively), $x$ is in a direction in the plane of the specimen, $z$ is in the direction of the incident electron beam, $V$ is the mean inner potential (MIP) of the specimen, and $B_{\perp}$ is the component of the magnetic induction perpendicular to both $x$ and $z$. For a sample with a thickness profile $t(x)$, when $V$ and $B_{\perp}$ are constant along $z$, Eq. (1) can be written as follows:

$$
\phi(\mathrm{x})=C_{\mathrm{E}} V_{0}(x) t(x)-\frac{e}{\hbar} \int B_{\perp}(x) t(x) d x .
$$

The gradient of the phase can be expressed by differentiating Eq. (2) with respect to $x$ to give

$$
\frac{d \phi(x)}{d x}=C_{\mathrm{E}} \frac{d}{d x}\left\{V_{0}(x) t(x)\right\}-\frac{e}{\hbar} B_{\perp}(x) t(x) .
$$

As can be seen from the above equations, in any phase image obtained from magnetic materials, both the in-plane magnetic field and the MIP contribute. To distinguish the magnetic contribution from that of the MIP, one can acquire holograms from each side of the specimen by turning it over, and then subtracting the two. The magnetic contribution to the phase shift changes sign while the MIP term stays unchanged. But a more practical method ${ }^{29}$ is to tilt the specimen by $\pm 30^{\circ}$ and switch on the objective lens at each angle to apply a magnetic field parallel to the beam direction. This then generates components of magnetic field along the axis (an in-plane component) and perpendicular to the axis of the specimen. Tilting the specimen with the same angle in two opposite directions generates two equal in-plane magnetic fields with opposite directions. Once the sample is magnetized in either direction, the objective lens is turned off and the specimen is tilted back to $0^{\circ}$ to record a hologram of the area of interest for each tilt. By taking the difference in the total phase shift from the two holograms, one obtains twice the magnetic contribution of the specimen to the phase shift.

The method described above relies on the assumption of having identical magnetic structures (with different magnetic signs) between the reversal experiments. For NWs with large aspect ratios $(t / r>1$, elliptical shape), complete reversal of magnetization is feasible. However, for NWs with smaller aspect ratios $(t / r<1$, disk-shape), the reversal pairs may not have identical magnetic structures. One needs to reverse each wire more than once to check the reproducibility of the measured phase shift. To calculate $B_{\perp}$ from Eq. (3), one needs the magnetic thickness of the specimen. Since this is likely to be smaller than the total (physical) thickness because of factors, such as surface oxidation, calculations based on the total thickness represent an upper limit on $B_{\perp}$.

\section{EXPERIMENTAL}

The NW arrays were electrodeposited into nanoporous alumina membranes that were obtained by a two-step anodization technique. ${ }^{30}$ The average pore diameter and interpore center-to-center distances were $40 \mathrm{~nm}$ and $110 \mathrm{~nm}$, respectively, as determined by scanning electron microscopy
(SEM), giving an average porosity of $10 \%$. Prior to deposition, a Ti adhesion layer $(15 \mathrm{~nm})$ and a Au layer $(1 \mu \mathrm{m})$ were sputtered onto the backside of the alumina membrane. The $\mathrm{Au} / \mathrm{Ti} /$ alumina film acted as the working electrode (cathode) in the electrochemical cell, and a platinum sheet was used as the counter electrode (anode) with a saturated calomel reference electrode. ${ }^{4}$ The NWs were grown inside the pores by pulsed-potential electrodeposition ${ }^{31}$ to produce quasihexagonal arrays of NWs. ${ }^{4,10}$ Multilayer NWs were fabricated using a single electrochemical bath by switching the cathode potential, $V$, between two values, $V_{F M}$ and $V_{N}(-1 \mathrm{~V}$ and $-0.56 \mathrm{~V}$, respectively), where $V_{F M}$ is the cathode potential used to deposit the ferromagnetic metal layer $(\mathrm{CoFeB})$ and $V_{N}$ the same for deposition of the nonmagnetic metal layer $(\mathrm{Cu})$. Since $V_{F M}$ is larger than $V_{N}, \mathrm{Cu}$ would also deposit during the deposition of the magnetic layer at $V_{F M}$. Therefore, the electrolyte $\mathrm{Cu}$ ion concentration was made more dilute compared with the magnetic metal ions to reduce its rate of incorporation into the magnetic layers.

The electrolyte used for the electrodeposition consisted of an aqueous solution of $\mathrm{CoSO}_{4} \cdot 6 \mathrm{H}_{2} \mathrm{O}(0.176 \mathrm{M}), \mathrm{FeSO}_{4} \cdot 6 \mathrm{H}_{2} \mathrm{O}$ $(0.03 \mathrm{M}), \mathrm{H}_{3} \mathrm{BO}_{3}(0.7 \mathrm{M}), \mathrm{CuSO}_{4} \cdot 5 \mathrm{H}_{2} \mathrm{O}(0.003 \mathrm{M}),\left(\mathrm{CH}_{3}\right)_{2}$ $\mathrm{NH}: \mathrm{BH}_{3}$, and $\mathrm{Na}$ saccharin $(0.005 \mathrm{M})$, at $\mathrm{pH}=3.5$. NW multilayers with nominal periodic non-magnetic/ferromagnetic layer thicknesses, $t_{\mathrm{N}} / \mathrm{t}_{\mathrm{FM}}$, of $7 / 9 \mathrm{~nm}, 10 / 50 \mathrm{~nm}$, and $75 / 75 \mathrm{~nm}$ and one tri-layer thickness geometry $t_{\mathrm{N}} / t_{\mathrm{FM}} / t_{\mathrm{N}} / t_{\mathrm{FM}}$ of $50 / 9 / 3 / 9 \mathrm{~nm}$, were studied. These layer thicknesses were estimated based on growth rates from constant-current electrodeposition of uniform composition NWs in a larger alumina membrane (pore diameter $170 \mathrm{~nm}$ ). An electrodeposition efficiency of $100 \%$ for the $\mathrm{Cu}$ layers and $70 \%$ for the CoFe layers was assumed. For STEM, wires were drop cast onto holey-carbon coated $\mathrm{Cu}$ grids after dissolving the nanoporous membranes in either sodium hydroxide ( $1 \mathrm{M}$ of $\mathrm{NaOH}, 6 \mathrm{~h}$ at room temperature) or phosphochromic acid $\left(0.5 \mathrm{M} \mathrm{H}_{3} \mathrm{PO}_{4}+0.2 \mathrm{M}\right.$ of $\mathrm{H}_{2} \mathrm{CrO}_{4}, 2 \mathrm{~h}$ at $70^{\circ} \mathrm{C}$ ).

The STEM was equipped with a field-emission source, operating at $200 \mathrm{keV}$ or $120 \mathrm{keV}$, an annular high-angle dark field detector, an energy-dispersive spectrometer (EDS), a rotatable electrostatic biprism, and a charge-coupled-device (CCD) camera. EH was carried out in a fixed beam TEM mode, while a scanning nanoprobe in STEM mode was used for compositional line maps via EDS analysis of x-ray emission. The microscope objective lens was turned off while the holograms were being acquired so that the NWs could be measured in a magnetic-field-free condition (less than 5 Gauss). Images were acquired via a Lorentz lens located below the sample. As discussed above, to separate the magnetic contribution from the MIP, we conducted an in situ magnetization reversal experiment by turning on the current of the objective lens while the sample was tilted. For each pair of holograms, the sample was tilted either $\pm 30^{\circ}$ (when $120 \mathrm{keV}$ was used) or $\pm 20^{\circ}$ (when $200 \mathrm{keV}$ was used). The NW orientation with respect to the tilt axis of the holder determined the magnitude and direction of the applied magnetic field. A biprism voltage of $140 \mathrm{~V}$ and a magnification of 42000 were used in Lorentz mode with an average magnetic resolution of $7.5 \mathrm{~nm}$ or $15 \mathrm{~nm}$ depending upon the microscope. The resolution of $\mathrm{EH}$ is equal to three times the 
holographic fringe spacing. ${ }^{26}$ Reference holograms were acquired from empty holes in the holey carbon-coated copper grids, away from any magnetic NWs. Holograms were recorded using the CCD camera. To provide quantitative information about magnetic properties, phase images were extracted from reconstructions of the holograms with the help of Sempar software. ${ }^{32}$

To help interpret the $B_{\perp}$ contour maps obtained from the holograms, magnetostatic simulations were performed using a commercial, finite-element software package. For these, a magnetic structure consisting of uniform magnetization with predetermined and identical orientation for all tetrahedral mesh cells was assumed within the magnetic layers. In other words, each magnetic layer was treated as a permanent magnet with predefined magnetization directions. The uniform magnetization values and the layer thicknesses for the simulations were taken as the average $B_{\perp}$ and $t$ measured by $\mathrm{EH}$ and STEM, respectively (Table I). A magnetic relative permeability, $\mu$ equal to unity was used for the non-magnetic layers, and as such, they had no influence on the field produced by the magnetic layers. The background was treated as vacuum, also with a $\mu$ of unity, and was increased in size until the boundaries had essentially no influence on the field produced by the ferromagnetic layers. The $B_{\perp}$ vector map was observed on a sagittal plane passing through the center of the wire.

\section{RESULTS}

A typical example of a bright field TEM (BF) image from a $\mathrm{Cu} / \mathrm{CoFeB}$ (nominally $10 / 50 \mathrm{~nm}$ ) multilayer NW is shown in Fig. 1(a) with the inset showing a selected area diffraction pattern (SAD). Phosphochromic acid was used to dissolve the alumina membrane in this case. No amorphous alumina debris is visible around the wire, but its surface is not smooth. The average diameter is $50 \mathrm{~nm}$. The $\mathrm{SAD}$ pattern indicates that both the $\mathrm{Cu}$ and $\mathrm{CoFeB}$ regions were random polycrystalline materials. Analysis of the ring diameters and spacing indicated the expected phases, face-centered cubic (FCC) $\mathrm{Cu}$ and bodycentered cubic (BCC) CoFeB (HRTEM images (not shown) showed grain sizes varying from $7 \mathrm{~nm}$ to $25 \mathrm{~nm}$ ).

The corresponding high-angle annular dark field STEM image of the same wire is shown in Fig. 1(b), where evidence

TABLE I. Summary of the NW samples investigated listing their periodic nonmagnetic $(\mathrm{Cu}) /$ ferromagnetic $(\mathrm{CoFeB}: \mathrm{Cu}=50: 50$ at. \%) layer thicknesses, $t_{N} / t_{F M}$, nominal values from uniform $\mathrm{NW}$ growth calibrations, and measured values from EDS in a STEM, and average magnetic induction, $B_{\perp}$, calculated from electron holography investigations assuming an average $\mathrm{NW}$ diameter of $50 \mathrm{~nm}$.

\begin{tabular}{|c|c|c|}
\hline \multicolumn{2}{|c|}{$\begin{array}{c}\mathrm{Cu} / \mathrm{CoFeB} \text { layer thicknesses } \\
t^{N} / t^{F M} \mathrm{~nm}\end{array}$} & \multirow{2}{*}{$\begin{array}{l}\text { Magnetic induction } \\
B_{\perp} \mathrm{T} \pm 0.1 \\
\text { Measured }\end{array}$} \\
\hline Nominal & Measured & \\
\hline $10 / 50$ & $50 / 50 \pm 5$ & 0.5 \\
\hline $75 / 75$ & $80 / 230 \pm 20$ & 1.0 \\
\hline $7 / 9$ & $12 / 8 \pm 3$ & 0.2 \\
\hline $50 / 9 / 3 / 9$ & $50 / 8 / 7 / 8 \pm 2$ & 0.3 \\
\hline
\end{tabular}

for the presence of multilayers is clear from the periodic contrast in the image. All layers are aligned with the axis of the wire. An EDS map along a line in the center of the same wire resulted in the integrated peaks from $\mathrm{Fe}, \mathrm{Co}$, and $\mathrm{Cu} \mathrm{X}$ ray emission, as shown in Fig. 1(c). The Co and Fe distributions are observed from the darker regions of the STEM image while those with predominantly $\mathrm{Cu}$ peaks are from the brighter regions. Contrast in this STEM image is proportional to the degree of scattering into the annular detector, primarily a function of sample mass. Since the density of $\mathrm{CoFe}$ and $\mathrm{Cu}$ is essentially identical, the variation in contrast is primarily due to the greater thickness apparent for the $\mathrm{Cu}$ layers compared with the $\mathrm{CoFeB}$, assuming a cylindrical geometry. The EDS profiles show that the FM/N interfaces are abrupt to within $5 \mathrm{~nm}, 10 \%-15 \%$ of the peak widths. However, $\mathrm{Cu}$ is found in the magnetic layers with a composition that varied from 5\% to $50 \%$ of the signal in the $\mathrm{Cu}$ layers. (A background signal from spurious electron interactions with the $\mathrm{Cu}$ support grids was subtracted.) The $\mathrm{B}$ component was not detected $(<2$ at. \%) by EDS. The nonmagnetic $\mathrm{Cu}$ layers were $50 \pm 5 \mathrm{~nm}$ thick along the axis, significantly greater than the nominal $10 \mathrm{~nm}$, and the same thickness as the magnetic layers. The Co:Fe composition ratio from EDS analysis was $2.7 \pm 0.5$ (x-ray emission crosssections for these elements are identical, to within the error).

A summary of the nominal and measured average thicknesses from similar STEM analyses of each sample investigated is shown in Table I. The Co/Fe ratio of the magnetic layers was always a similar value, on average 3 , and a significant $\mathrm{Cu}$ composition (50 at. \%) was present in all samples. The measured thickness of individual layers was often greater than the nominal estimate by different factors that were highly nonlinear. In particular, when the expected layers were thinner than the NW diameter $(50 \mathrm{~nm})$, both $\mathrm{Cu}$ and $\mathrm{CoFeB}$ tended to be thicker than expected. For layer thicknesses larger than the diameter, the $\mathrm{CoFeB}$ layers tended to be thicker, while $\mathrm{Cu}$ grew at the expected rate. These discrepancies indicate that pulsed-potential electrodeposition of $\mathrm{CoFeB}$ and $\mathrm{Cu}$ was on average faster in the smaller pore diameter membranes $(50 \mathrm{~nm})$ compared with the rates anticipated from larger pore $(170 \mathrm{~nm})$ constant current calibrations.

Figures 2 and 3 show results from EH on NWs from the same sample as shown in Fig. 1, as a function of the influence of the direction of an applied, external magnetic field on the remnant magnetic structure of the layers. Fig. 2(a) shows a hologram obtained at $120 \mathrm{keV}$ and Fig. 2(b) shows the magnetic contribution to the phase shift, represented by contours, reconstructed from the hologram in (a), after a magnetic field of $1 \mathrm{~T}$ was applied parallel to the wire axis, and then removed. Each contour in this map, and all subsequent images, represents a phase shift of 0.6 radians, equivalent to a $0.1 \mathrm{~T}$ change in $B_{\perp}$, calculated from Eq. (3), assuming an average NW diameter of $50 \mathrm{~nm}$. We observe that the $B_{\perp}$ contours are almost exactly aligned with the axis of the wire with a radial deviation at both ends of a magnetic layer. Since the aspect ratio of each layer is larger than 1 , we expect to see such an out-of-plane flower state for these lines. We attribute the small deviations in alignment $\left(<5^{\circ}\right)$ 


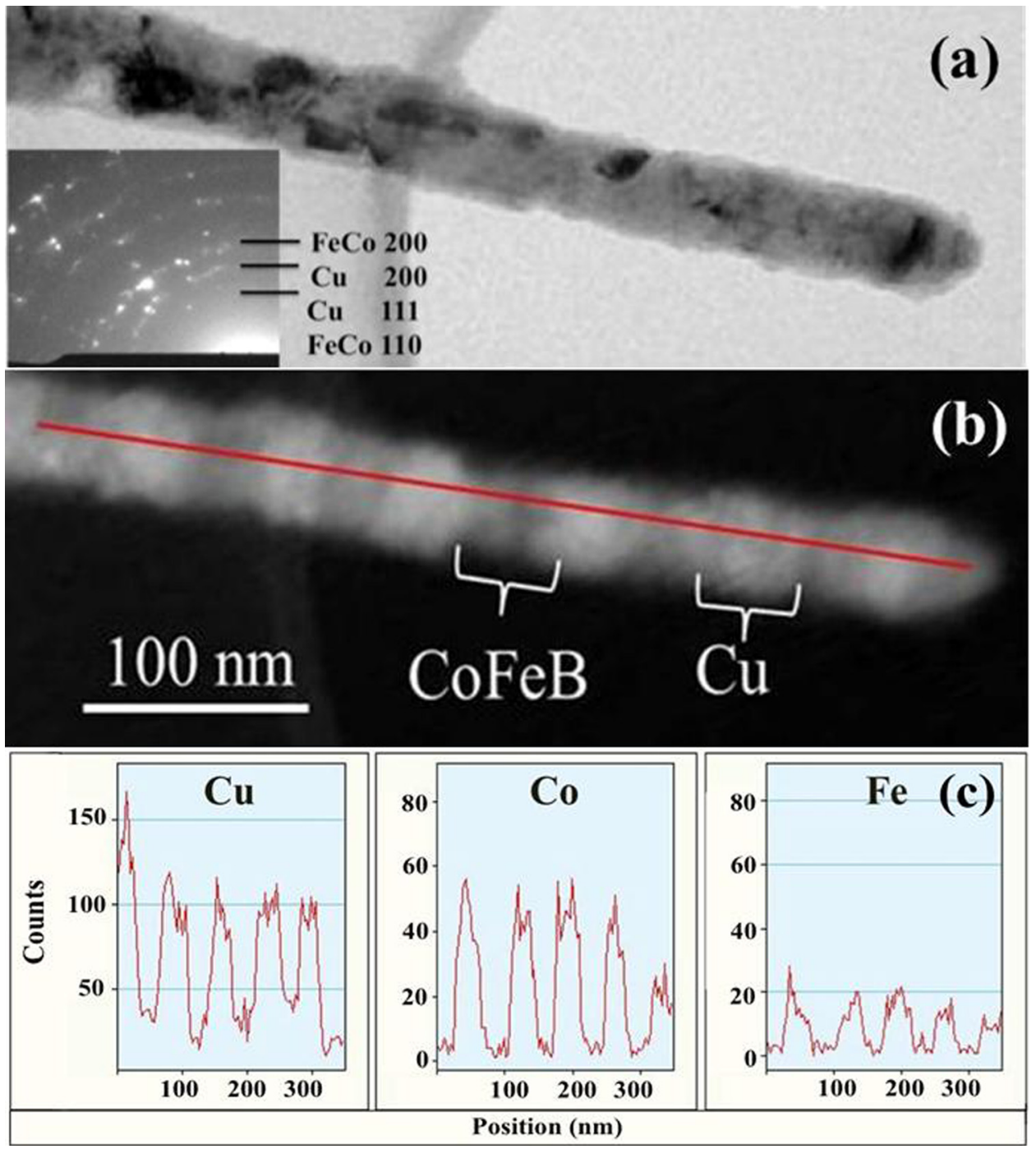

FIG. 1. (a) Bright field TEM image and SAD pattern (inset) and (b) STEM image (acquired at $200 \mathrm{keV}$ ) of a $\mathrm{Cu} / \mathrm{CoFeB}(50 / 50 \mathrm{~nm}) \mathrm{NW}$. (c) Plots of the integrated peak count from EDS along the axis of the same wire (red line in (b)) from $\mathrm{Cu}, \mathrm{Co}$, and $\mathrm{Fe}$ spectra.

to local demagnetizing fields of a given layer and to the effect on a given layer of its magnetic neighbours. The average total $B_{\perp}$ of the magnetic layers is $0.5 \mathrm{~T}$ as determined from the number of magnetic contours in each layer (5). The magnetostatic simulation of the $B_{\perp}$ vector map, in Fig. 2(c), assumes a uniform longitudinal magnetization in each magnetic layer and displays the expected direction of the $B_{\perp}$ contour lines seen in the hologram.

Similarly, Figs. 3(a) and 3(b) show a hologram $(120 \mathrm{keV})$, and the magnetic contribution of the reconstructed hologram, but after the application of an external field perpendicular to the axis. In this case, we expect that each layer will be in an in-plane flower state. This is what is observed in some parts of Fig. 3(b) (white boxed areas 1 and 2 ) where the $B_{\perp}$ contour lines are at an angle between that of the wire axis and the direction of the external field applied before the measurement. Fig. 3(c) shows the simulated $B_{\perp}$ vector map with the magnetization of the magnetic layers alternating between $+45^{\circ}$ and $-45^{\circ}$ relative to the wire axis.

An example of a hologram and its $B_{\perp}$ contour map, from a NW with larger layer thicknesses $(80 / 230 \mathrm{~nm})$, is shown in Figs. 4(a) and 4(b) $(120 \mathrm{keV})$. The magnetic field was applied parallel to the NW axis. In this case, $\mathrm{NaOH}$ was used to dissolve the alumina membrane. Alumina debris on the surface of the NW is apparent from the image, based on the lower contrast and irregular shape of this amorphous material. The average NW diameter is $50 \mathrm{~nm}$ but the surface of the wire is not smooth. Based on the $B_{\perp}$ contour lines of Fig. 4(b) (spacing $0.1 \mathrm{~T}$ ), it is clear that the magnetization is along the axis of the NW as expected, with an average total $B_{\perp}$ of $1 \mathrm{~T}$.

A hologram and $B_{\perp}$ contour map of another NW of the same sample are shown in Figs. 5(a) and 5(b) (120 keV) after an external magnetic field had been applied at an angle of $45^{\circ}$ to the axis. Again, as expected from shape anisotropy, the magnetization inside the wires is uniform and follows the shape of the wire. The $B_{\perp}$ contour line spacing is $0.1 \mathrm{~T}$, indicating a total average measured $B_{\perp}$ in the magnetic layers, of $1 \mathrm{~T}$. The magnetostatic simulations in Figs. 4(c) and 5(c) produce corresponding $B_{\perp}$ vector maps assuming axially magnetized layers.

Figure 6(a) shows an example of a STEM image, obtained at $200 \mathrm{keV}$, from a $\mathrm{Cu} / \mathrm{CoFeB}(12 / 8 \mathrm{~nm}) \mathrm{NW}$ that was dissolved in $\mathrm{NaOH}$. Like other samples (Figs. 4 and 5), 

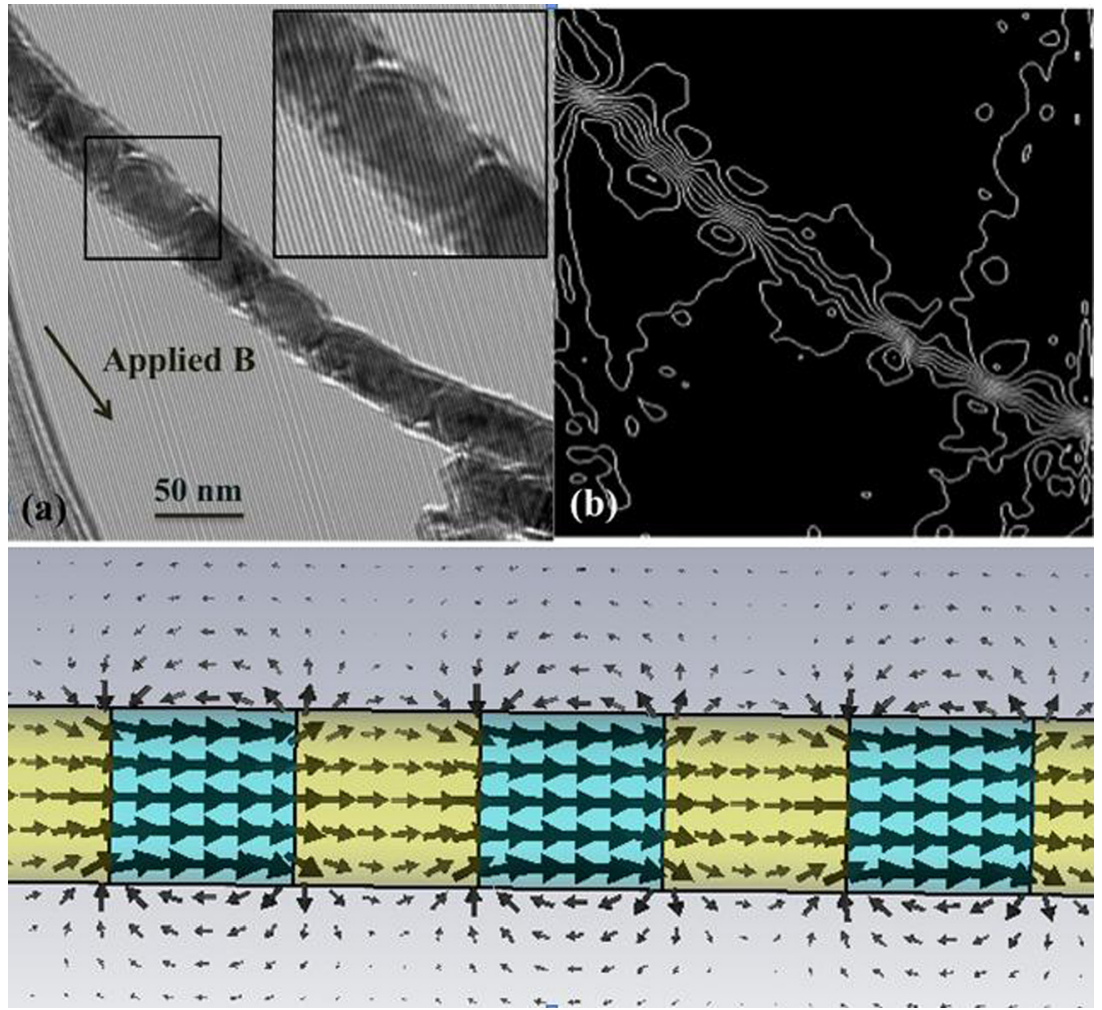

FIG. 2. (a) Hologram (acquired at $120 \mathrm{keV}$ ) and (b) associated remnant $B_{\perp}$ map of a $\mathrm{Cu} / \mathrm{CoFeB}(50 / 50 \mathrm{~nm})$ NW for a magnetic field applied parallel to the axis of the NW prior to the hologram acquisition. The contour spacing is $0.1 \mathrm{~T}$. Note that there is a missing magnetic layer in the middle of image. (c) Magnetostatic simulation of the $B_{\perp}$ vector map with axially magnetized magnetic layers (blue represents the $\mathrm{CoFeB}$ layer and yellow represents the $\mathrm{Cu}$ layer).

the presence of alumina debris on the wire is evident. The NW in Fig. 6(a) has a relatively smooth surface and multiple layers are present. Note that some of the layer boundaries have small deviations in orientation with respect to the axis of the NW, as indicated by an arrow. Fig. 6(b) shows an EDS map along the red line at the left corner of the same $\mathrm{NW}$, which has resolved the layer $\mathrm{x}$-ray emission from Fe,
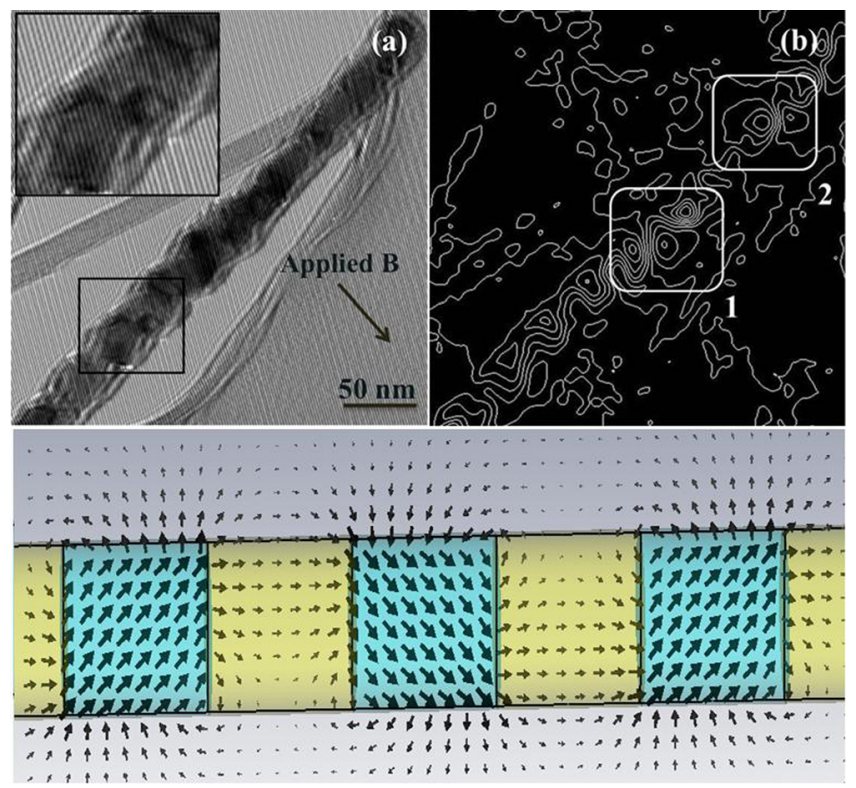

FIG. 3. (a) Hologram (acquired at $120 \mathrm{keV}$ ) and (b) associated $B_{\perp}$ map of a $\mathrm{Cu} / \mathrm{CoFeB}(50 / 50 \mathrm{~nm}) \mathrm{NW}$ after a magnetic field had been applied perpendicular to the axis of the wire. The contour spacing is $0.1 \mathrm{~T}$. (c) Magnetostatic simulation of the $B_{\perp}$ vector map with the magnetization of the magnetic layers alternating between $+45^{\circ}$ and $-45^{\circ}$ relative to the wire axis (blue represents the $\mathrm{CoFeB}$ layer and yellow represents the $\mathrm{Cu}$ layer).
$\mathrm{Co}$, and $\mathrm{Cu}$. The $\mathrm{Co}$ and $\mathrm{Fe}$ peaks clearly indicate a layer thickness of $8 \pm 2 \mathrm{~nm}$ for the magnetic layer, whereas the $\mathrm{Cu}$ profile follows the periodicity of the Fe and Co peaks, with a thickness of $12 \pm 3 \mathrm{~nm}$. The average spatial resolution of the magnetic phase achievable with this electron microscope (200 keV) was $12 \mathrm{~nm}$ (with a fringe spacing of $4 \mathrm{~nm}$ ). Therefore, it was not possible to detect the magnetic signals from these smaller individual magnetic layers, unless the effect of a combination of layers was present in the magnetic phase images.

Figure 7 shows (a) a hologram (200 keV) and (b) a magnified selection of the same hologram, its MIP phase contribution, magnetic phase contribution, and $B_{\perp}$ contour map of another $\mathrm{Cu} / \mathrm{CoFeB}(12 / 8 \mathrm{~nm}) \mathrm{NW}$ in a parallel applied magnetic field. The magnetic phase image (Fig. 7(b)) shows a small phase change across the NW, associated with the magnetic signal from the trilayers. The $B_{\perp}$ contour map shows lines parallel to the axis of the NW which can be interpreted as a $B_{\perp}$ signal from a combination of layers along this part of the NW. No $B_{\perp}$ signal was detected from the hologram of such NWs for a perpendicular applied magnetic field, probably due to limited spatial resolution. The magnetostatic simulation of the $B_{\perp}$ vector map is shown in Fig. 7(c), where $B_{\perp}$ is expected to be concentrated within the wire due to the proximity of the axially magnetized layers.

Figures 8(a) and 8(b) show a hologram and associated $B_{\perp}$ contour map from a sample consisting of a linear array of periodic $\mathrm{FM} / \mathrm{N} / \mathrm{FM}$ tri-layers separated by large $\mathrm{Cu}$ spacer layers, (50/8/7/8 nm) NW, after magnetization in a perpendicular applied magnetic field. The NW diameter is again $50 \mathrm{~nm}$ and the magnetic resolution of this microscope is $7.5 \mathrm{~nm}$ (three times the fringe spacing of $2.5 \mathrm{~nm}$ ). Hence, a magnetic signal from individual layers was not detected. In 

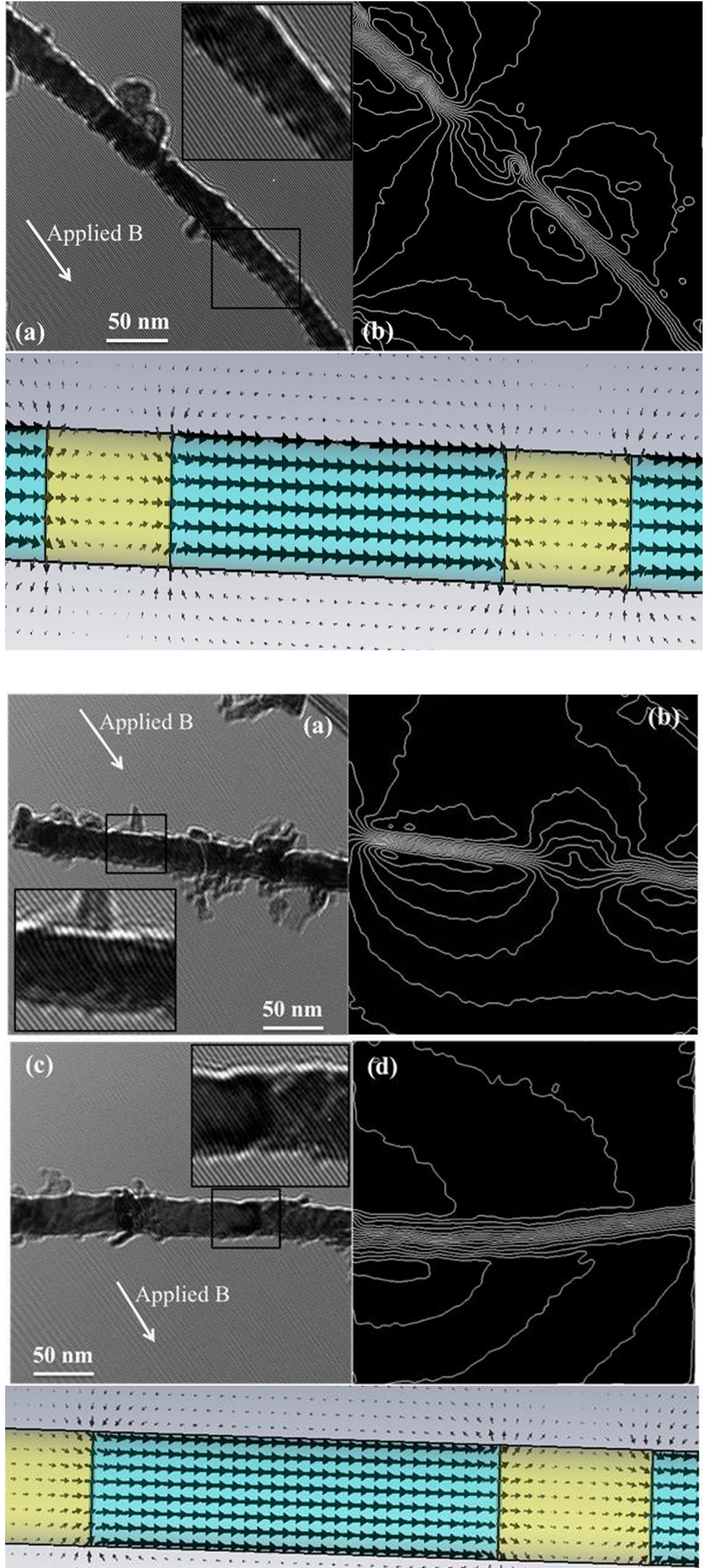

FIG. 4. (a) Hologram (acquired at $120 \mathrm{keV}$ ) and (b) associated $B_{\perp}$ map of a $\mathrm{Cu} / \mathrm{CoFeB}(80 / 230 \mathrm{~nm}) \mathrm{NW}$ for a magnetic field applied parallel to the axis of the NW. The contour spacing is $0.1 \mathrm{~T}$. (c) Magnetostatic simulation of the $B_{\perp}$ map with axially magnetized magnetic layers. The apparent vortex state in the nonmagnetic layer is due to artifacts from image processing (blue represents the $\mathrm{CoFeB}$ layer and yellow represents the $\mathrm{Cu}$ layer).

FIG. 5. (a) Hologram (acquired at $120 \mathrm{keV}$ ) and (b) $B_{\perp}$ map of a $\mathrm{Cu} /$ $\mathrm{CoFeB}(80 / 230 \mathrm{~nm}) \mathrm{NW}$ for an applied magnetic field with a $45^{\circ}$ angle with respect to the axis of each wire. The contour spacing is $0.1 \mathrm{~T}$. (c) Magnetostatic simulation of the $B_{\perp}$ map with an axially magnetized magnetic layers (blue represents the $\mathrm{CoFeB}$ layer and yellow represents the $\mathrm{Cu}$ layer). 

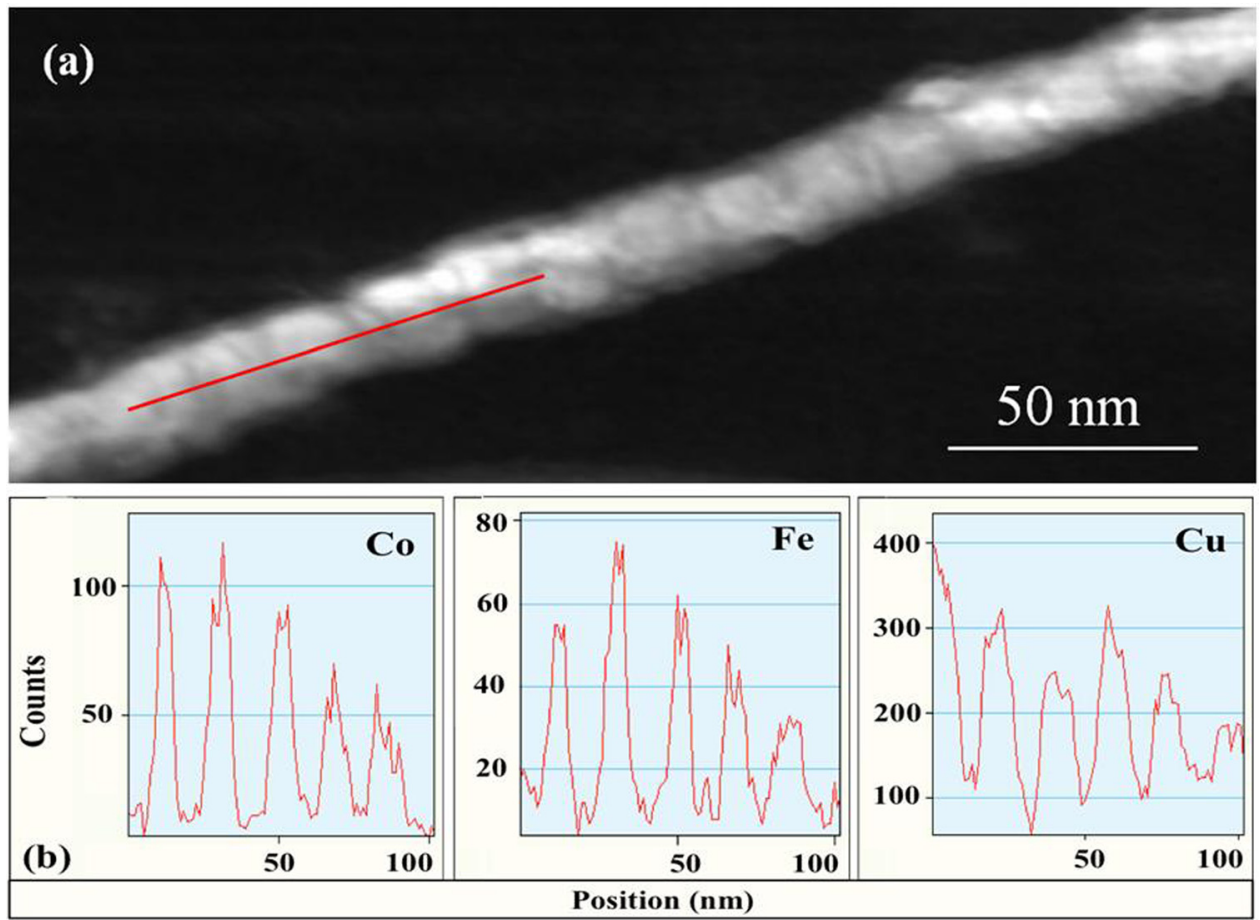

FIG. 6. (a) STEM image (acquired at $200 \mathrm{keV}$ ) of a $\mathrm{Cu} / \mathrm{CoFeB}(12 / 8 \mathrm{~nm}) \mathrm{NW}$ and (b) plots of the integrated peak count from EDS collected along the axis of the same NW in (a) (red line) from $\mathrm{Cu}, \mathrm{Co}$, and $\mathrm{Fe}$ spectra.

addition, there was no signal detected in the $50 \mathrm{~nm} \mathrm{Cu}$ spacer layers between the tri-layers due to magnetic interaction between the combined layers along the NW. We would expect to see opposing magnetization in the neighbouring magnetic layers in this sample because of dipolar interactions. A signal from a combination of the tri-layers through their dipolar interactions might appear as a vortex. In fact, such a signal is observed at the bottom-left corner of Fig. 8(b) (white boxed area), where the size of the vortex is consistent with the tri-layer geometry. Different holograms acquired from the same type of NW showed a similar signal. No $B_{\perp}$ signal was detected from the rest of the NW, and the feature in the middle of the hologram is a phase unwrapping artifact (a conclusion that is based on comparisons of multiple holograms obtained from same region). Fig. 8(c) shows a magnetostatic simulation with the magnetizations of the two magnetic layers in a tri-layer anti-parallel, as expected for the strong dipolar coupling between these layers.

Finally, Figs. 9(a) and 9(b) show an example of a hologram (200 keV) from the NW of Fig. 8 , and its $B_{\perp}$ contour

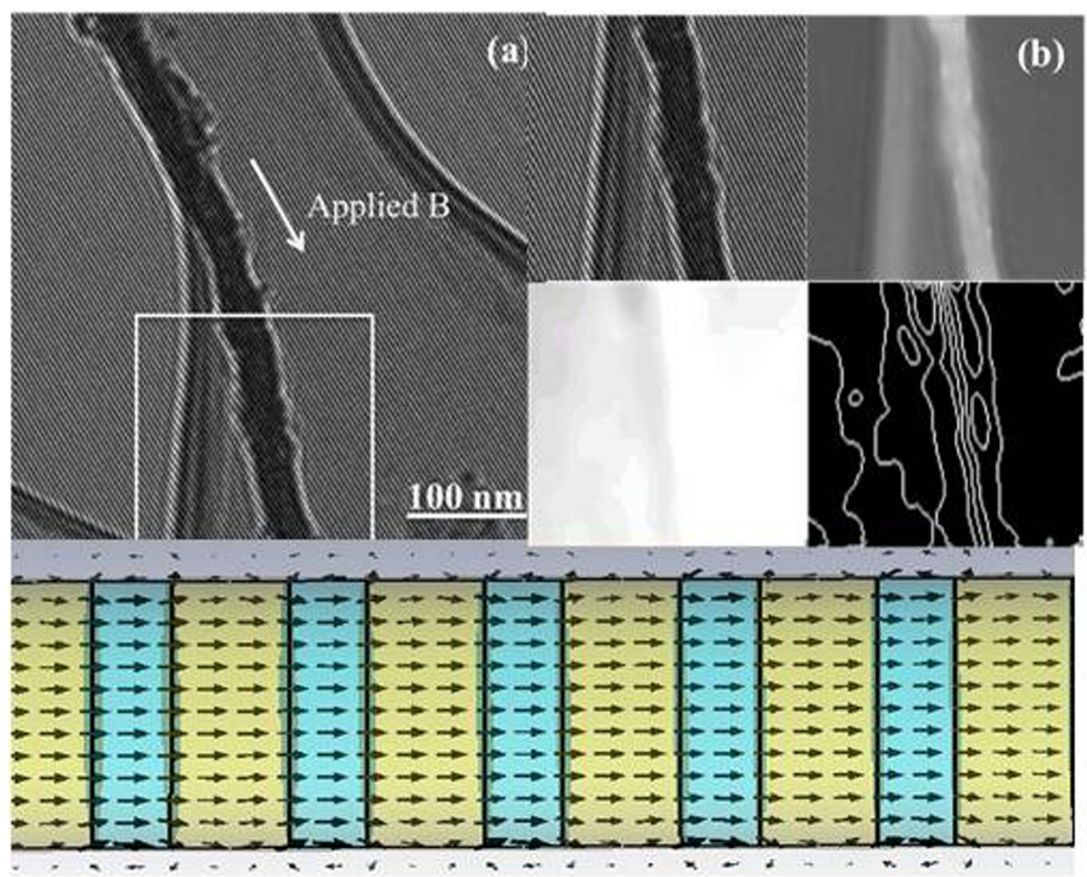

FIG. 7. (a) Hologram (acquired at $200 \mathrm{keV}$ ) and (b) selected area of (a) with its associated MIP phase contribution, magnetic phase contribution, and $B_{\perp}$ map of a $\mathrm{Cu} / \mathrm{CoFeB}(12 / 8 \mathrm{~nm}) \mathrm{NW}$ for a magnetic field applied parallel to the axis of the wire. The contour spacing is $0.1 \mathrm{~T}$. (c) Magnetostatic simulation of the $B_{\perp}$ vector map of the same geometry (blue represents the $\mathrm{CoFeB}$ layer and yellow represents the $\mathrm{Cu}$ layer). 


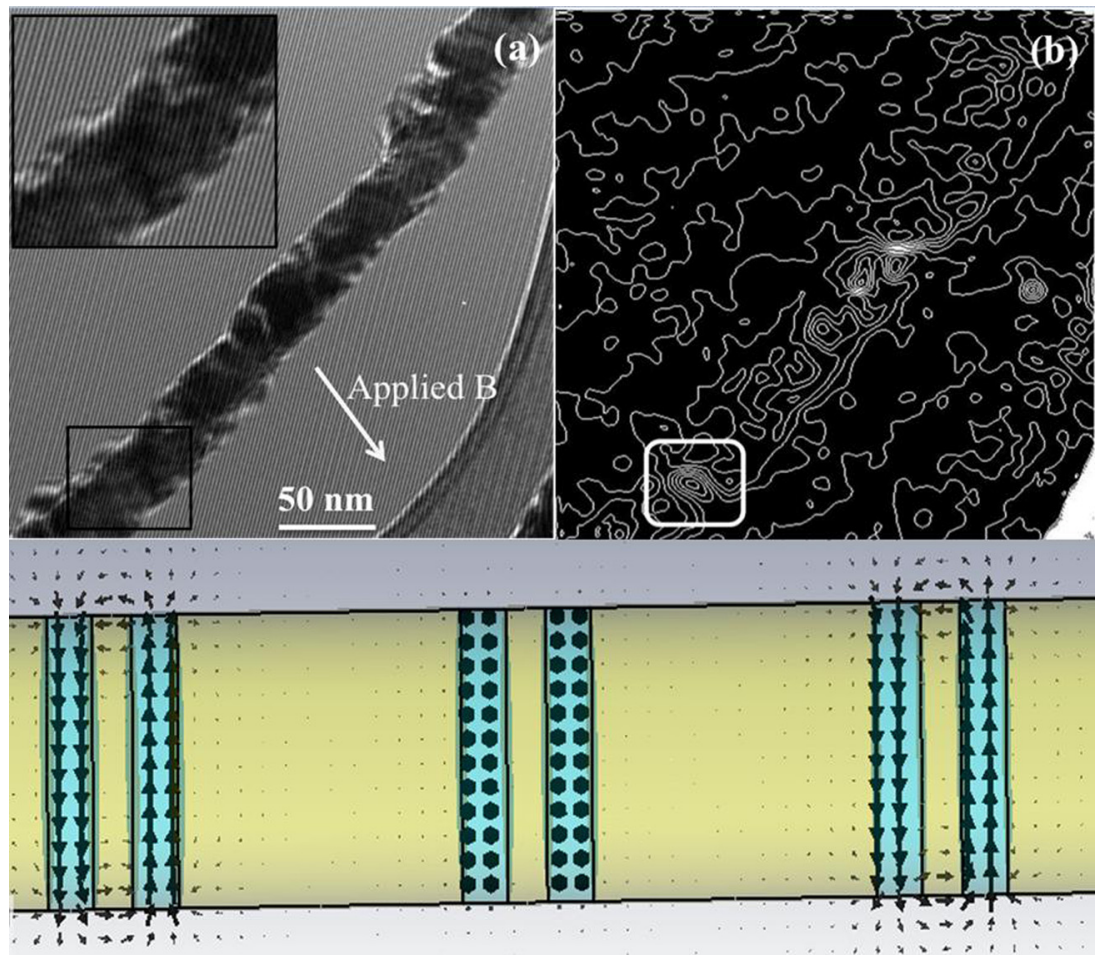

FIG. 8. (a) Hologram (acquired at $120 \mathrm{keV}$ ) and (b) associated $B \perp$ map of a $\mathrm{Cu} / \mathrm{CoFeB} / \mathrm{Cu} / \mathrm{CoFeB}(50 / 8 / 7 / 8 \mathrm{~nm})$ NW for a perpendicular applied magnetic field. The white rectangular area shows an opposite orientation of magnetization in the neighbouring layers (an apparent magnetic vortex). (c) Magnetostatic simulation of the $B_{\perp}$ map. The magnetization of each set of tri-layers is anti-parallel, with an arbitrary azimuthal alignment (blue represents the $\mathrm{CoFeB}$ layer and yellow represents the $\mathrm{Cu}$ layer).

map, respectively, for an applied magnetic field parallel to the axis of the NW. Again, detection of a magnetic signal is limited by the spatial resolution $(12 \mathrm{~nm})$. Most of the $B_{\perp}$ contour lines in Fig. 9(b) are in fact reconstruction artifacts except for the lines in the indicated area (white box). In this region, there is a detectable magnetic signal that is consistent with a $(30 \mathrm{~nm})$ tri-layer, with $B_{\perp}$ at the same angle to the axis of NW in both layers. The magnetostatic simulation of the $B_{\perp}$ vector map, Fig. 9(c), shows a NW with the likely anti-parallel alignment for each set of tri-layers consistent with the contour map. The magnetization of both sets of magnetic layers of the middle tri-layer is oriented at $30^{\circ}$ relative to the wire axis.

\section{DISCUSSION}

The contribution of the dipolar interaction to the effective magnetic anisotropy of an array of multilayered NWs critically depends upon the chemical composition and the thicknesses of the magnetic layers relative to the nonmagnetic spacer layers. Using Eq. (8) of Ref. 10, and assuming vanishing porosity to model the case of a single wire, the

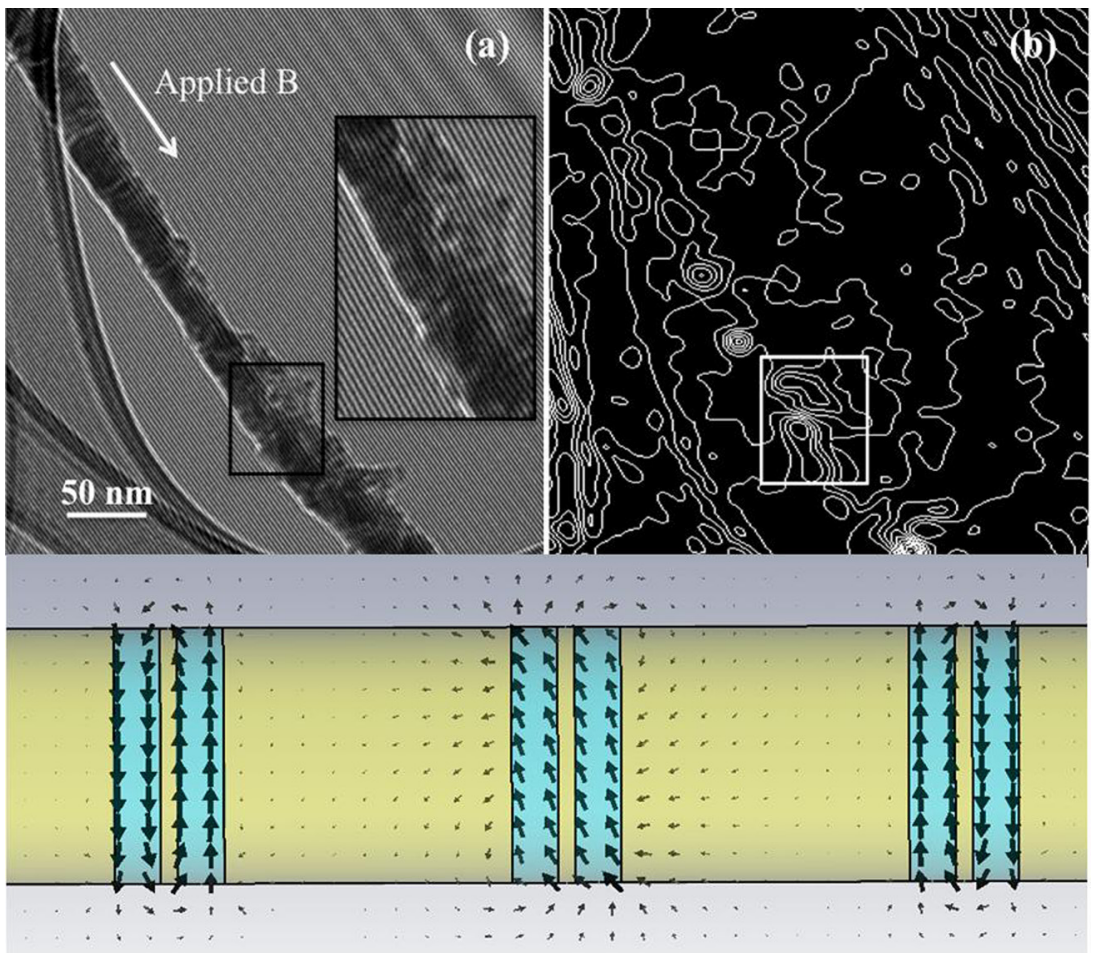

FIG. 9. (a) Hologram (acquired at $200 \mathrm{keV}$ ) and (b) associated $B_{\perp}$ map of a $\mathrm{Cu} / \mathrm{CoFeB} / \mathrm{Cu} / \mathrm{CoFeB}(50 / 8 / 7 / 8 \mathrm{~nm})$ NW for a parallel applied magnetic field. The white rectangular area shows the magnetic contour map corresponding to the effect of a tri-layer with an angle to the axis of the NW. (c) Magnetostatic simulation of the $B$ vector map, with anti-parallel alignment for each set of tri-layers. The magnetization of both magnetic layers of the middle tri-layer is oriented at $30^{\circ}$ relative to the wire axis (blue represents the $\mathrm{CoFeB}$ layer and yellow represents the $\mathrm{Cu}$ layer). 
effective dipolar anisotropy constant can be expressed as follows:

$$
\begin{aligned}
K_{e f f} & =\frac{\mu_{0} M_{s}^{2}}{2}\left[\frac{3 f}{2}-1\right]=\frac{\mu_{0} M_{s}^{2}}{2}\left[\frac{3 / 2}{1+t_{N} / t_{F M}}-1\right] \Rightarrow B_{e f f} \\
& =\mu_{0} M_{s}\left[\frac{3 / 2}{1+t_{N} / t_{F M}}-1\right]
\end{aligned}
$$

where $f$ is the relative thickness of the magnetic layer to the total thickness of magnetic plus non-magnetic layers (or equivalently, the relative fraction of magnetic material in a single $\mathrm{NW}$ ), and $\mu_{0} M_{s}$ is the saturation magnetization. The anisotropy constant can be associated with an effective anisotropy field, $B_{\text {eff }}=2 K_{\text {eff }} / M_{\mathrm{s}}$, parallel to the wire axis. As expected, for large $t_{\mathrm{N}} / t_{F M}$ ratios, the value in the bracket is -1 , corresponding to an anisotropy of a thin cylinder, dominated by the $-\mu_{0} M_{\mathrm{s}}$ demagnetizing field. In such circumstances, the remanent magnetization (assumed uniform in this simplified model) will be randomly oriented perpendicular to the wire axis. For small $t_{\mathrm{N}} / t_{F M}$ ratios, the bracket is $1 / 2$, corresponding to an equivalent anisotropy field of $\mu_{0} M_{\mathrm{s}} / 2$ parallel to the wire axis. It is important to emphasize that even thin ferromagnetic layers can exhibit an out-of-plane remanent state due to the dipolar interaction with other ferromagnetic layers, provided that the $\mathrm{Cu}$ spacers are much thinner than the ferromagnetic layers.

Equation (4) provides a general understanding of the effect of the saturation magnetization, $\mu_{0} M_{\mathrm{s}}$, and of $t_{\mathrm{N}} / t_{\mathrm{FM}}$, on the effective dipolar anisotropy field. A summary of the measured values for the transverse magnetic induction is found in Table I. Both the $\mathrm{Cu}$ and $\mathrm{CoFeB}$ layers were nanocrystalline (FCC and BCC, respectively) based on SAD patterns. There was no evidence that the $\mathrm{CoFeB}$ was amorphous as reported for $\mathrm{Co}_{94} \mathrm{Fe}_{5} \mathrm{~B}_{1}$ thin films and single composition NW arrays. ${ }^{31}$ The NW surfaces were rougher and there was more evidence of alumina debris present when the template was dissolved in $\mathrm{NaOH}$, compared with phosphochromic acid. But when alumina debris was present, it did not apparently affect the EH measurement or sensitivity.

Our off-axis EH technique has been able to resolve magnetic volumes as small as $\left(20 \mathrm{~nm}^{3}\right)$ using a single biprism in a field-emission electron column at $120 \mathrm{keV}$. The measured magnetic induction at technical saturation of a thin film of $\mathrm{Co}_{94} \mathrm{Fe}_{5} \mathrm{~B}_{1}$ electrodeposited on a gold-coated substrate under conditions similar to those employed for wire deposition was $1.7 \pm 0.1 \mathrm{~T}$, in agreement with the bulk value of $1.6 \mathrm{~T}{ }^{33}$ The $\mathrm{CoFeB}$ layers all exhibited average $\mathrm{Co} / \mathrm{Fe}$ ratios of approximately 3 , for which the bulk value would be also $1.7 \mathrm{~T}$, if they contained no $\mathrm{Cu}$. However, increasing $\mathrm{Cu}$ content rapidly decreases this value, which ranged between $1 \mathrm{~T}$ and $0.5 \mathrm{~T}$ for thicknesses between $230 \mathrm{~nm}$ and $50 \mathrm{~nm}$, and was even smaller for thinner layers. Since the multilayers had ferromagnetic-nonmagnetic interfacial thicknesses of $5 \mathrm{~nm}$, it is to be expected that thin layers would have higher average $\mathrm{Cu}$ content, and lower magnetization, as observed.

For relatively thick magnetic layers compared with the $\mathrm{Cu}$ layers, the magnetic contour lines followed the shape of the NWs, parallel to the axis of the NW with small demagnetization effects clearly evident in the $\mathrm{Cu}$ interlayers $(80 \mathrm{~nm})$. From Eq. (4), the threshold above which a ferromagnetic layer starts to be dominated by its self demagnetizing field is $t_{\mathrm{N}} / t_{F \mathrm{M}}>2$. That is, except for the $\mathrm{Cu} /$ $\mathrm{CoFeB}=80 / 230 \mathrm{~nm}$ sample, the FM layers are expected to experience stronger self-demagnetization fields than the "remagnetizing" dipolar fields of the other layers. Hence, the measured $B_{\perp}$ values for the $50 / 50$ and $80 / 230 \mathrm{~nm}$ samples ( 0.5 and $1.0 \mathrm{~T}$, respectively) indicate a greater influence of dipolar coupling in the smaller thickness CoFeB. Again this assumes that the $\mathrm{Cu}$ composition remains high in both samples.

For thinner layers, a deviation in the direction of the average magnetic induction of individual magnetic layers, away from exactly parallel to the axis, was indeed observed. This is attributed to demagnetizing effects within the layers and due to the influence of magnetic neighbours. While the magnetic anisotropy appears to be dominated by the dipolar interaction, one cannot rule out other contributions. Notably, the observation of the off-axis remanence in the contour lines of the NWs magnetized by perpendicular fields suggests also a contribution to the total anisotropy from a magnetocrystalline anisotropy.

Finally, it is important to realize that Eq. (4), which assumes a uniform magnetization in each layer, neglects the possibility of vortex or flower states in individual layers, as discussed in the introduction. In fact, it may come as a surprise to some that the effective anisotropy, estimated from Eq. (4), is independent of the ratio of the thickness to the diameter of each individual layer. That conclusion is due to the long range nature of the dipolar interaction, and to the implicit assumption that the multilayered NW is significantly longer than it is wide (regardless of the ratio of individual layers). For the tri-layered samples, however, the situation is different. There, the strong dipolar coupling between the neighbouring magnetic layers leads to an anti-parallel configuration of the pairs within a trilayer, effectively screening out any long range dipolar field from the other sets of trilayers. For the smallest thickness layers $(8 / 7 / 8 \mathrm{~nm})$, magnetic signals associated with the interaction of neighbouring layers were detected.

\section{CONCLUSIONS}

Crystallographic and magnetic properties of isolated electrodeposited ferromagnetic multilayer NWs $(\mathrm{Cu} / \mathrm{CoFeB})$ were investigated, using off-axis EH and analytical STEM techniques. Several nonmagnetic/ferromagnetic layer periods were studied, including average thicknesses of $50 / 50 \pm 5 \mathrm{~nm}$, $80 / 230 \pm 20 \mathrm{~nm}, 12 / 8 \pm 3 \mathrm{~nm}$, and $8 / 7 / 8 / 50 \pm 2 \mathrm{~nm}$, as determined by STEM and EDS analysis. The composition of the magnetic $\mathrm{CoFeB}$ layers was $3 / 1 \mathrm{Co} / \mathrm{Fe}$ with up to $50 \% \mathrm{Cu}$. The $\mathrm{B}$ was below the level of sensitivity of the EDS $(<2$ at. \%). The $\mathrm{CoFeB}$ and $\mathrm{Cu}$ layers were both found to be crystalline rather than the expected amorphous magnetic phase. The presence of a significant $\mathrm{Cu}$ in the $\mathrm{CoFeB}$ likely had a strong influence on the anticipated magnetic anisotropy, consistent with results from macroscopic measurements of similar arrays. ${ }^{10}$

The observed magnetization in the wires from $\mathrm{EH}$ agreed with our expectations based on the magnetic layer 
aspect ratios (thickness to radius) and the expected dipolar fields of the neighbouring layers. The magnetization direction in the wires was predominantly parallel to the longer dimension of the magnetic volumes and was relatively uniform throughout the magnetic layer. However, depending upon the thicknesses of the layers, the behavior could differ. An angle between the magnetization and the axis of the wire was observed for magnetic layers thinner than the diameter $(50 \mathrm{~nm})$. The lower maximum induction values $(0.2-1 \mathrm{~T})$ compared with expected bulk values for $\mathrm{CoFeB}$ alloys $(1.7 \mathrm{~T})$ are most likely the result of the high $\mathrm{Cu}$ content in the NWs (50 at. \%).

STEM images of $\mathrm{Cu} / \mathrm{CoFeB}(50 / 50 \mathrm{~nm}) \mathrm{NW}$ s confirmed that these wires were multilayered with non-magnetic/ferromagnetic interfacial thicknesses of $5 \mathrm{~nm}$. EH showed that an applied external field available via the objective lens could control the remanent magnetization direction. As expected, this could be parallel to the axis of the wire (applied external magnetic field parallel to the wire axis) or at an angle to the axis (applied field perpendicular to the axis). It is probable that magnetocrystalline anisotropy played a primary role in producing these remanent states and contributed to the small variations in the crystals seen by waviness in their magnetic induction contours. The NWs with the largest thicknesses $\mathrm{Cu} / \mathrm{CoFeB}(80 / 230 \mathrm{~nm})$ showed a magnetic induction whose direction followed the wire axis independent of the angle of the external field. In this case, the dipolar field dominated the magnetization in all circumstances.

Electron holography from thinner multilayers of $\mathrm{Cu}$ / $\mathrm{CoFeB}(12 / 8 \mathrm{~nm})$ and a tri-layer sample $\mathrm{Cu} / \mathrm{CoFeB} / \mathrm{Cu} /$ $\mathrm{CoFeB}(50 / 8 / 7 / 8 \mathrm{~nm})$ in NWs with $50 \mathrm{~nm}$ diameters did not provide a detectable magnetic signal from individual layers. While the existence of these layers was confirmed from EDS profiling, their individual thicknesses generated a magnetic induction signal that was presumably below the detection limit of the technique. However, for both types of NWs, vortex-shaped magnetic signals, associated with a combination of layers, were observed. These vortices apparently formed within the thin tri-layers from the expected opposing orientations of dipolar-coupled magnetization. Considering that the dipolar effect from the neighbouring tri-layers $(50 \mathrm{~nm}$ distant $)$ would be negligible, instead the tri-layers behaved roughly like quadrupoles with a much smaller interaction range.

\section{ACKNOWLEDGMENTS}

We are grateful for partial funding of this work by the Natural Science and Engineering Research Council of Canada. We thank Dr. S. Yazdi for help with microscopy at DTU.
${ }^{1}$ C. A. Ross, H. I. Smith, T. Savas, M. Schattenburg, M. Farhoud, M. Huang, M. Walsh, M. C. Abraham, and R. J. Ram, J. Vac. Sci. Technol. B 17, 3168 (1999).

${ }^{2}$ T. Shimizu, K. Aoki, Y. Tanaka, T. Terui, and S. Shingubara, Jpn. J. Appl. Phys. Part 1 50, 06GE01 (2011).

${ }^{3}$ A. Fert and L. Piraux, J. Magn. Magn. Mater. 200, 338 (1999).

${ }^{4}$ L. P. Carignan, A. Yelon, D. Ménard, and C. Caloz, IEEE Trans. Microwave Theory Tech. 59, 2568 (2011).

${ }^{5}$ D. H. Reich, M. Tanase, A. Hultgren, L. A. Bauer, C. S. Chen, and G. J. Meyer, J. Appl. Phys. 93, 7275 (2003).

${ }^{6}$ M. Tanase, D. M. Silevitch, A. Hultgren, L. A. Bauer, P. C. Searson, G. J. Meyer, and D. H. Reich, J. Appl. Phys. 91, 8549 (2002).

${ }^{7}$ T. Thurn-Albrecht, J. Schotter, G. A. Kästle, N. Emley, T. Shibauchi, L. Krusin-Elbaum, K. Guarini, C. T. Black, M. T. Tuominen, and T. P. Russell, Science 290, 2126 (2000).

${ }^{8}$ S. S. P. Parkin, M. Hayashi, and L. Thomas, Science 320, 190 (2008).

${ }^{9}$ P. P. Pal and R. Pati, Phys. Rev. B 77, 144430 (2008).

${ }^{10}$ L. P. Carignan, C. Lacroix, A. Ouimet, M. Ciureanu, A. Yelon, and D. Ménard, J. Appl. Phys. 102, 023905 (2007).

${ }^{11}$ A. Saib, M. Darques, L. Piraux, D. Vanhoenacker-Janvier, and I. Huynen, IEEE Trans. Microwave Theory Tech. 53, 2043 (2005).

${ }^{12}$ C. A. Ross, Annu. Rev. Mater. Sci. 31, 203 (2001).

${ }^{13}$ M. Darques, J. Spiegel, J. De la Torre Medina, I. Huynen, and L. Piraux, J. Magn. Magn. Mater. 321, 2055 (2009).

${ }^{14}$ V. Boucher, L.-P. Carignan, T. Kodera, C. Caloz, A. Yelon, and D. Ménard, Phys. Rev. B 80, 224402 (2009).

${ }^{15}$ L.-P. Carignan, M. Massicotte, C. Caloz, A. Yelon, and D. Ménard, IEEE Trans. Magn. 45, 4070 (2009).

${ }^{16}$ I. Mayergoyz, Mathematical Models of Hysteresis and Their Applications (Elsevier, Amsterdam, 2003).

${ }^{17}$ F. Béron, L. Clime, M. Ciureanu, D. Ménard, R. W. Cochrane, and A. Yelon, J. Nanosci. Nanotech. 8, 2944 (2008).

${ }^{18}$ F. Béron, L.-P. Carignan, D. Ménard, and A. Yelon, in Electrodeposited Nanowires and Their Applications, edited by N. Lupu (INTECH, Croatia, 2010).

${ }^{19}$ F. Béron, L.-P. Carignan, D. Ménard, and A. Yelon, IEEE Trans. Magn. 44, 2745 (2008).

${ }^{20}$ C. A. Ross, M. Hwang, M. Shima, J. Y. Cheng, M. Farhoud, T. A. Savas, H. I. Smith, W. Schwarzacher, F. M. Ross, M. Redjdal, and F. B. Humphrey, Phys. Rev. B 65, 144417 (2002).

${ }^{21}$ C. Beeli, B. Doudin, J. P. Ansermet, and P. A. Stadelmann, Ultramicroscopy 67, 143 (1997).

${ }^{22}$ E. Snoeck, R. E. Dunin-Borkowski, F. Dumestre, P. Renaud, C. Amiens, B. Chaudret, and P. Zurcher, Appl. Phys. Lett. 82, 88 (2003).

${ }^{23}$ R. E. Dunin-Borkowski, T. Kasama, A. Wei, S. L. Tripp, M. J. Hytch, E. Snoeck, R. J. Harrison, and A. Putnis, Microsc. Res. Technol. 64, 390 (2004)

${ }^{24} \mathrm{H}$. Lichte, Ultramicroscopy 38, 13-22 (1991).

${ }^{25}$ A. Tonomura, Adv. Phys. 41, 59 (1992).

${ }^{26}$ R. E. Dunin-Borkowski, M. R. McCartney, and D. J. Smith, Electron Holography of Nanostructured Materials, Encyclopaedia of Nanoscience and Nanotechnology (American Scientific Publishers, 2004), Vol. 3, p. 41.

${ }^{27}$ M. Gajdardziska-Josifovska, M. R. McCartney, W. J. de Ruijter, D. J. Smith, J. K. Weiss, and J. M. Zuo, Ultramicroscopy 50, 285 (1993).

${ }^{28}$ L. Reimer, Transmission Electron Microscopy (Springer, 1991).

${ }^{29}$ R. E. Dunin-Borkowski, M. R. McCartney, D. J. Smith, and S. S. P. Parkin, Ultramicroscopy 74, 61 (1998).

${ }^{30}$ S. Zhao, K. Chan, A. Yelon, and T. Veres, Nanotechnology 18, 245304 (2007).

${ }^{31}$ M. Ciureanu, F. Béron, L. Clime, P. Ciureanu, A. Yelon, T. A. Ovari, R. W. Cochrane, F. Normandin, and T. Veres, Electrochim. Acta 50, 4487 (2005).

${ }^{32}$ W. O. Saxton, T. J. Pitt, and M. Horner, Ultramicroscopy 4, 343 (1979).

${ }^{33}$ R. M. Bozorth, Ferromagnetism (Wiley IEEE Press, Piscataway, NJ, 1978). 